University of Nebraska - Lincoln

DigitalCommons@University of Nebraska - Lincoln

March 1969

\title{
Local Moment Formation and Resistivity Minima in CoAl Alloys
}

\author{
G.R. Caskey \\ Center for Materials Science and Engineering, Massachusetts Institute of Technology, Cambridge, \\ Massachusetts
}

David J. Sellmyer

University of Nebraska-Lincoln, dsellmyer@unl.edu

Follow this and additional works at: https://digitalcommons.unl.edu/physicssellmyer

Part of the Physics Commons

Caskey, G.R. and Sellmyer, David J., "Local Moment Formation and Resistivity Minima in CoAl Alloys" (1969). David Sellmyer Publications. 183.

https://digitalcommons.unl.edu/physicssellmyer/183

This Article is brought to you for free and open access by the Research Papers in Physics and Astronomy at DigitalCommons@University of Nebraska - Lincoln. It has been accepted for inclusion in David Sellmyer Publications by an authorized administrator of DigitalCommons@University of Nebraska - Lincoln. 


\title{
Local Moment Formation and Resistivity Minima in CoAl Alloys*
}

\author{
G. R. Caskey and D. J. Sellmyer \\ Center for Materials Science and Engineering, Massachusetts Institute of Technology, Cambridge, Massachusetls 02139
}

\begin{abstract}
Measurements of electrical resistivity, thermoelectric power, and magnetoresistance have been made from $1.4^{\circ}$ to $300^{\circ} \mathrm{K}$ in alloys of $48 \%-57 \%$ cobalt in aluminum. At the equiatomic composition, CoAl has the $\mathrm{CsCl}$ structure. Resistance minima were observed in the vicinity of $30^{\circ} \mathrm{K}$ for cobalt concentrations between $50.4 \%$ and $51.5 \%$. Below the resistance minima there is a temperature region where the dependence is approximately logarithmic before it saturates at about $1.4^{\circ} \mathrm{K}$. The depth of the minima $\left[\rho\left(1.4^{\circ} \mathrm{K}\right)-\rho\left(T_{\mathrm{min}}\right)\right]$ and the thermopower at $4.2^{\circ} \mathrm{K}$ both show rather sharp peaks as a function of concentration in the vicinity of $50.5 \%$ cobalt. The magnetoresistance of the samples showing minima is negative or $T<T_{\min }$ and its magnitude increases with field up to $150 \mathrm{kG}$. These results and earlier susceptibility measurements for this system are discussed in terms of several current theories of the electronic effects of magnetic impurities in metals.
\end{abstract}

There have been many observations of anomalies in the low-temperature electronic properties of metals with small concentrations of magnetic impurities. In the present work, similar effects are reported in concentrated alloys of cobalt in aluminum. The cobalt concentration $c$, in atomic percent, ranged from 48-57. At the equiatomic composition the alloy has the ordered $\mathrm{CsCl}$ structure which is simple cubic with the aluminum and cobalt atoms at $\left(\frac{1}{2}, \frac{1}{2}, \frac{1}{2}\right)$ and $(0,0,0)$ positions, respectively. It is known that for $57 \gtrsim c>50$, cobalt atoms replace aluminum atoms substitutionally whereas for $47 \lesssim c<50$, the cobalt deficiency is achieved through vacancies on cobalt sites. ${ }^{1}$ The interesting magnetic effects occur for $c$ slightly larger than 50 so that, for this concentration range, the alloy might be regarded as a dilute solid solution of cobalt atoms in the ordered metallic compound CoAl.

\section{EXPERIMENTAL RESULTS}

Figure 1 shows the temperature dependence of the resistance ratio, $r(T) \equiv R(T) / R\left(4.2^{\circ} \mathrm{K}\right)$, for $49 \lesssim c \lesssim$ 51.9. Specimens in the range $50.4 \leq c \leq 51.5$ exhibit a resistance minimum at a temperature $T_{\min }$ in the vicinity of $30^{\circ} \mathrm{K}$. Both $T_{\min }$ and the depth of the minimum are dependent upon composition. Except for $\mathrm{Co}(50.4), r(T)$ has reached a constant value at $1.4^{\circ} \mathrm{K}$. The temperature dependence of $r(T)$ for the samples showing a minimum is approximately logarithmic in the middle portion of the interval between $1.4^{\circ} \mathrm{K}$ and $T_{\min }$.

The transverse magnetoresistance $(\Delta \rho / \rho)$, measured between $1.6^{\circ}$ and $77^{\circ} \mathrm{K}$, is negative at all fields up to $150 \mathrm{kG}$ for alloys with $c>50$. For $\mathrm{Co}(49.0)$ the magnetoresistance is positive except at the lowest fields and for all samples, the magnitude does not saturate at $150 \mathrm{kG}$.

* This research was supported by the Advanced Research Projects Agency and was performed in part at the Francis Bitter National Magnet Laboratory which is supported by the U.S.A.F.O.S.R.

1 N. Ridley, J. Inst. Metals 94, 255 (1966).
Figure 2 shows the composition dependence of the thermoelectric power at $4.2^{\circ}$ and $296^{\circ} \mathrm{K}$. The rather sharp structure exhibited, in particular, at $4.2^{\circ} \mathrm{K}$ occurs for the same compositions that show resistivity minima. The sample that shows the strongest resistance minimum, Co(50.4), also shows a positive peak in the thermopower at $7^{\circ} \mathrm{K}$ which is roughly at the midpoint of the resistivity rise for this specimen.

The susceptibility of this alloy system was measured at $5 \mathrm{kG}$ between $77^{\circ}$ and $300^{\circ} \mathrm{K}$ by West. ${ }^{2}$ In this temperature range, $\chi$ is essentially independent of temperature for $c=49.4$. For $c=50.8$ and 51.2, $\chi$ follows an antiferromagnetic Curie-Weiss law, and for $52.8 \leq$ $c \leq 54.5$ a ferromagnetic Curie-Weiss law. The magnetization of specimen $\mathrm{Co}(50.4)$ was measured at $4.2^{\circ} \mathrm{K}$ in fields up to $50 \mathrm{kG}^{3}$ and was proportional to $(\Delta \rho / \rho)^{1 / 2}$. The susceptibility was a decreasing function of field and the limiting value of $d \sigma / d H$ was $9.4 \times 10^{-6}$ $\mathrm{emu} / \mathrm{g}$ at $50 \mathrm{kG}$.

\section{DISCUSSION}

In light of the temperature and concentration dependence of $\chi$, and the sign of the magnetoresistance, it may be hypothesized that for $c \leq 50$ there are no localized moments and that for $c$ slightly larger than 50, magnetic moments exist on the excess cobalt atoms. This hypothesis seems reasonable since, for $c<50$ none of the cobalt atoms has nearest neighbors which are not aluminum atoms. However, for $c>50$ the excess cobalt atoms exist at the center of a cube with eight nearestneighbor cobalt atoms at the corners, a structure that would seem to be conducive to the formation of a local moment on the excess cobalt atoms.

In view of the approximate logarithmic temperature dependence of the resistivity, it is natural to ask whether the data can be explained in terms of the Kondo effect ${ }^{4}$

\footnotetext{
${ }^{2}$ G. W. West, Phil. Mag. 15, 855 (1967).

${ }^{3}$ These measurements were made by $\dot{\mathrm{S}}$. Foner and I. De Grave.

${ }^{4} \mathrm{~J}$. Kondo, Progr. Theoret. Phys. 32, 37 (1964).
} 
in which the excess cobalt atoms would have to be considered as the "impurity" atoms in the "pure" metallic compound. If we take this point of view and let the atomic percent of the impurity atoms be denoted by $x \equiv c-50$ for $c \geq 50$, then the Kondo theory would imply that $\rho_{\text {spin }} \propto x$. Taking $\rho_{\text {spin }}=\rho(T)-\rho\left(T_{\min }\right)$ gives $\rho_{\mathrm{spin}} / x=$ constant for $x=0.4$ and 0.6 but not for larger $x$. For larger $x$, interactions between the impurity atoms would presumably become important and, in fact, the susceptibility data suggest that the alloy becomes ferromagnetic at low temperatures between $52 \%$ and $53 \%$ cobalt.

There are, however, other theories which may be relevant to this system. For example, the theory of localized spin fluctuations of Rivier et al., ${ }^{5}$ predicts a weak resistance minimum with a low-temperature logarithmic dependence, antiferromagnetic Curie-Weiss behavior in the susceptibility, and a tendency towards a giant thermopower as the lifetime of the spin fluctuations becomes large. Clearly, our data on the depth of the resistance minimum $\left[\rho\left(1.4^{\circ} \mathrm{K}\right)-\rho\left(T_{\min }\right)\right]$ as well as the thermopower at $4.2^{\circ} \mathrm{K}$ show sharp peaks at a concentration of about $50.5 \%$ cobalt. If localized spin

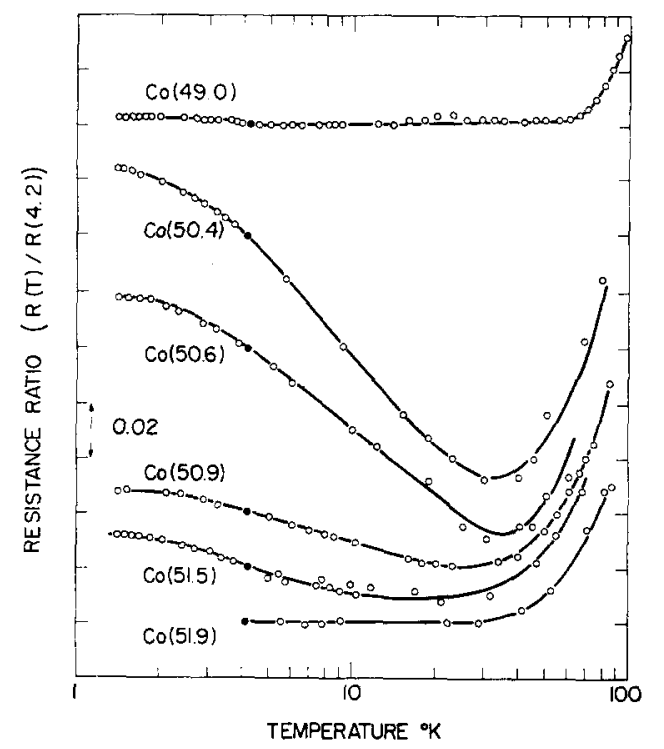

FIG. 1. Temperature dependence of the resistance ratio $R(T) / R(4.2)$. The vertical placement of the curves is arbitrary but the solid points show where the ratio is defined to be unity.

${ }^{5}$ N. Rivier, M. J. Zuckerman, and M. Sunjic (unpublished); see also: D. J. Kim, Phys. Rev. 146, 455 (1966).

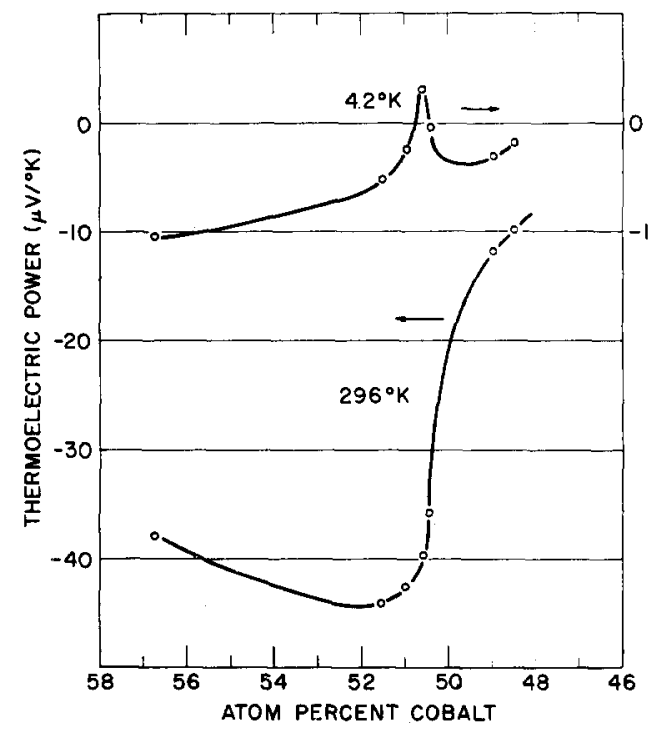

FIG. 2. Thermoelectric power vs composition.

fluctuations are important in this system, then this would suggest that $c \simeq 50.5$ is the critical concentration at which the impurity states approach the magnetic limit.

A further possible explanation, perhaps, is that localized moments exist not only on the excess cobalt atoms, but also on their eight nearest-neighbor cobalt atoms. From the susceptibility data in the range $50.8<c<53$, one can derive an effective magnetic moment of 8.9 Bohr magnetons per excess cobalt atom. If, in fact, the neighbors of the impurity cobalt atoms are magnetic, then it would be necessary to consider the interaction of the conduction electrons with interacting impurity pairs or even multiple spin complexes. Dekker, Brailsford and Overhauser, and others have considered the interaction of the conduction electrons with coupled impurity spins and this theory also can predict a resistance minimum and negative magnetoresistance under certain circumstances. ${ }^{6}$ Obviously, a detailed interpretation of these results will have to rely on further low-temperature susceptibility data and also microscopic magnetic measurements.

We wish to thank Dr. D. J. Kim for several helpful discussions.

${ }^{6}$ For a review of these theories, see: T. Van Peski-Tinbergen and A. J. Dekker, Physica 29, 917 (1963). 